\title{
MODELO DE GESTIÓN DE LA DEMANDA ENERGÉTICA INTEGRAL*
}

\author{
ENRIQUE HURTADO AGUIRRE*** \& JUAN PABLO ESCAMILLA MEJÍA*** \\ UNIVERSIDAD MILITAR NUEVA GRANADA - UNIVERSIDAD NACIONAL DE COLOMBIA
}

Recibido/ Received/ Recebido: 21/07/14 - Aceptado/ Accepted / Aprovado: 16/06/15

\begin{abstract}
Resumen
La gestión energética significa optimizar una de las más complejas e importantes técnicas creaciones que conocemos: el sistema de energía. Existen una buena cantidad de métodos para la optimización de la generación de energía y su distribución. Por lo que el modelo de la demanda energética integral (DSM) es una estrategia para mejorar el sistema de energía en el lado del consumo. Se extiende desde la política energética, la economía experimental, la teoría de los agentes, la mejora de la eficiencia energética mediante software de soporte y aplicación, las tarifas de energía inteligente con incentivos para ciertos patrones de consumo, hasta los sofisticados sistemas de control en tiempo real de los recursos energéticos distribuidos. Este documento proporciona una visión general y una taxonomía para el desarrollo de un modelo sistemático integral de DSM, que analiza los distintos elementos de construcción, y ofrece un panorama sobre cómo emplear un modelo de análisis jerárquico de procesos y evaluación integral difusa para su implementación.

Palabras clave: Análisis de proceso jerárquico (AHP); Gestión de la demanda; Modelo del sistema eléctrico; Ingeniería Inteligente; Software de soporte y aplicación; Análisis difuso.
\end{abstract}

\section{INTEGRAL ENERGY DEMAND MODEL MANAGEMENT}

\begin{abstract}
Energy management means optimizing one of the most complex and important technical creations that we know: the power system. There are a lot of methods for optimization of power generation and distribution. So the model of comprehensive energy demand (DSM) is a strategy to improve the energy system in the consumption side. It extends from energy policy, experimental economics, the theory of agents, improved energy efficiency through support and application software, intelligent power rates with incentives for certain consumption patterns to sophisticated control systems realtime distributed energy resources. This document provides an overview and a taxonomy for the
\end{abstract}

\footnotetext{
Artículo resultado del Proyecto de Investigación ECO 1219, denominado: "Retos para la implementación de un modelo de gestión de proyectos de eficiencia en la demanda de energía eléctrica en Colombia". Financiado la Vicerrectoría de Investigaciones de la UMNG. ECO 1219 de 2013. Proyecto desarrollado en el Grupo de Estudios en Ciencias Económicas (CIE) de la Universidad Militar Nueva Granada.

** Magister en Administración de la Universidad Nacional de Colombia. Docente de la Universidad Nacional de Colombia y Universidad Militar. Líder e Investigador tiempo completo Grupo CIE, Facultad de Ciencias Económicas, Universidad Militar Nueva Granada, Bogotá, Colombia. Dirección Postal: Cra. 11 \# 101-80, Bloque C, Piso 1. Bogotá (Colombia). Teléfono: 6500000 Ext. 1306. Correo electrónico: enrique.hurtado@unimilitar.edu.co.

**** Docente investigador facultad de ingeniería de la Universidad Nacional de Colombia. Correo electrónico: jpescamillam@unal.edu.co.
} 
development of a comprehensive systematic model of DSM, which analyzes the different elements of construction, and provides a perspective of how to use a model of process hierarchical analysis and blurred comprehensive evaluation for implementation.

Keywords: Hierarchical Process Analysis (AHP); Demand management; Electric System Model; Intelligent Engineering; Support and Implementation Software; Blurred Analysis.

\title{
MODELO DE GERENCIAMENTO DA DEMANDA ENERGÉTICA INTEGRAL
}

\begin{abstract}
Resumo
A gerência energética busca otimizar uma das mais complexas e importantes técnicas que conhecemos: o sistema de energia. Existe uma boa quantidade de métodos para a otimização da geração de energia e sua distribuição. O modelo da demanda energética integral (DSM) é uma estratégia para melhorar o sistema de energia no lado do consumo. Estende-se desde a política energética, a economia experimental, a teoria dos agentes, a melhora da eficiência energética mediante software de suporte e aplicativo, as tarifas de energia inteligente com incentivos para certos padrões de consumo, até os sofisticados sistemas de controle em tempo real dos recursos energéticos distribuídos. Este documento proporciona uma visão geral e uma taxonomia para o desenvolvimento de um modelo sistemático integral de DSM, que analisa os diferentes elementos de construção e oferece um panorama sobre como empregar um modelo de análise hierárquica de processos e avaliação integral difusa para sua implementação.

Palavras chave: Análises de processo hierárquico (AHP); Gerenciamento da demanda; Modelo do sistema elétrico; Engenharia Inteligente; Software de suporte e aplicativo; Análise difusa.
\end{abstract}

Hurtado, E. \& Escamilla, J. (2015). Modelo de gestión de la demanda energética integral. En: Revista de la Facultad de Ciencias Económicas de la Universidad Militar Nueva Granada. rev.fac.cienc. econ, XXIII (2), DOI: http://dx.doi.org/10.18359/rfce.1612.

JEL: Q41, L52.

\section{Introducción}

La Gestión centrada en el modelo de la demanda energética integral (DSM, por sus siglas en ingles) y un modelo integral de gestión de la demanda de electricidad (GDE), es un reto de trabajo de ingeniería de desarrollo e ingeniería eléctrica, que involucra a muchos participantes, denominados usualmente como agentes a saber: los gobiernos, las empresas de la red eléctrica, empresas de generación de energía, empresas de servicios de ahorro de energía y los usuarios de energía (Boyle, 1996). Adicionalmente, vincula la planificación, creación, diseño, implementación, evaluación ex post de los proyectos, etc. Estos agentes tienen diferentes objetos e intereses respectivamente, y la especialización del conocimiento en cada vínculo es comparativamente más fuerte, en aspectos tales como: el sistema de energía eléctrica, la economía industrial, la protección del medio ambiente, el derecho, etc.

Esta situación genera una gran cantidad de dificultades, una intensa labor y retos para el normal desarrollo de la DSM como es la consulta de datos, el procesamiento, análisis y cálculo, entre otros; si alguno de estos procesos se hace manualmente, el costo sería una gran cantidad de tiempo, esfuerzo y dinero. Afortunadamente el desarrollo de las tecnologías de información, hardware y software se ha ido aplicando ampliamente en campos como la consulta de datos, transmisión, almacenamiento, análisis y procesamiento. Al valerse de la tecnología, un modelo de desarrollo, el trabajo del administrador de la demanda podrá establecer una plataforma de apli- 
caciones, que resolvería problemas tediosos como el procesamiento de datos, el intercambio de información entre otros, proporcionando así un fuerte apoyo para el proceso de desarrollo (Hamdan, Ghajar, \& Chedid, 2012).

Además del procesamiento monótono de datos, también se requiere del apoyo informático, para la evaluación de las políticas y diversas medidas del DSM. La gestión centrada en la demanda es empleada de manera científica y eficaz, para estimar con anticipación los posibles efectos generados después de la aplicación de los procedimientos y disposiciones de la GDE.

A menudo es difícil referirnos a normativas y programaciones históricas, debido a que pueden ser estas medidas completamente nuevas y no tener ningún punto de referencia. Para ser evaluadas requieren de un avance en el tiempo y ser debidamente formulados posterior a su aplicación, ya que podrían ocasionar una gran pérdida a la economía y a la sociedad. Una gestión bien concebida podría reducir los picos de demanda en un mercado que funcione correctamente (Räsänen, Ruusunen \& Hämäläinen, 1995).

Para ello es necesario hacer varios experimentos para observar sus diferentes resultados, como ocurre en las ciencias duras o exactas, de esta manera, podemos encontrar problemas a tiempo y evitar pérdidas. Sin embargo, el trabajo del DSM es un problema social y económico que es diferente a uno en ciencias naturales. ¿Cómo hacer experimentos con este tipo de problema? Marx pensaba que no podemos usar un cuchillo o tubo de ensayo químico para investigar una cuestión social y económica, una forma es emplear un método para modelar con la capacidad abstracta del hombre y llevar a cabo diversos experimentos de simulación a través del soporte de software y hardware (Wei \& Liang, 2009).

Es por esto, que es valioso crear un modelo integral de gestión de la demanda de electricidad, que no sólo puede mejorar la eficiencia del trabajo en el desarrollo de la DSM, sino que también es beneficioso para el proceso científico de establecer las políticas y medidas del GDE.

\section{Elementos clave para la implementa- ción del modelo GDE}

\subsection{Economía experimental}

El llamado experimento económico es una forma empírica que se aplica en la investigación económica. Específicamente, lo que se hace es cambiar parte del entorno objeto de estudio, mediante reglas o el control de algunas condiciones (hipótesis), luego se observa el comportamiento de este objeto experimental, se analiza el resultado en un entorno controlable, para inspeccionar, comparar y completar la teoría económica, facilitar la política y la base de decisión apuntando a un cierto fenómeno económico (Chen, Wang, Wang \& Chen, 2004). La base teórica de un experimento económico, se considera en un sistema micro-económico que incluye dos partes a saber, "Entorno" y "Sistema". El "Entorno" se utiliza para describir las características de los sistemas económicos, enfatizando en los elementos de estudio de manera general, incluyendo los atributos del agente (económico), como son: la preferencia, la tecnología, el conocimiento y la condición inicial, etc. El "Sistema", tiene una composición comparativamente más complicada y se describe desde la reglamentación del agente económico en el sistema económico definido por el "Entorno", que incluye principalmente: las reglas del lenguaje, reglas de distribución de costos, las normas de reglamentación, etc.(Elshkaki, Van Der Voet, Van Holderbeke \& Timmermans, 2004).

A través del método de la economía experimental, existen cinco tipos de trabajo que se pueden desarrollar (Brañas-Garza \& Espinosa, 2011) a saber:

i. La comprobación teórica: mediante un experimento se realiza la comparación de la información enviada por el objeto experimental comprobando si los resultados pueden verificar una teoría. Si el grado de similitud del valor observado es consistente con el valor esperado teórico y/o es mayor, se puede asegurar que este tipo de consistencia no es causado por el azar y entonces podemos decir que es una teoría verificada.

ii. Búsqueda de causas para la teoría de ineficiencia: una vez que el resultado observado en el 
experimento es diferente de la teoría y si el diseño experimental no viola la hipótesis teórica, se puede considerar que la teoría tiene un problema; entonces, la causa específica de la teoría de ineficiencia se puede encontrar mediante el diseño de experimentos equivalentes y pertinentes.

iii. El establecimiento de reglas experimentales como base para una nueva teoría: en un experimento económico, los teóricos pueden investigar condiciones un tanto complicadas con facilidad y observar el resultado experimental con el fin de obtener las normas experimentales para guiar el establecimiento de una nueva teoría.

iv. Comparación del entorno: en un mismo sistema, se puede modificar el entorno experimental de los resultados antes y después del cambio, para comparar las influencias de los diferentes entornos (tales como la oferta y demanda estructural) en el resultado.

v. Comparación del sistema: bajo un mismo entorno experimental, en el resultado, las influencias de diferentes sistemas se comparan cambiando el sistema experimental (como la estructura de la información, las reglas de transacción, etc.).

En el siglo XX, y debido al gran desarrollo de la productividad, aparecieron muchos problemas de gran tamaño y retos que la tecnología y la ingeniería de sistemas, basados en la investigación operativa, la cibernética, la teoría de la información, y las ciencias de la administración, se perfilaron dando su solución en un momento determinado. La descripción y solución de estos problemas verosímiles, en su mayoría son modelos matemáticos abstractos. Sin embargo, existe un gran número de causalidades que no se pueden expresar mediante ecuaciones matemáticas o representaciones físicas, como son los grafos de relaciones que requieren ampliar su significado en la forma que las tratamos actualmente (Huang et al., 2011).

\subsection{Ingeniería inteligente}

El modelo generalizado $\mathrm{F}: \rightarrow \mathrm{X}$, que describe los grafos de relaciones, se aplica en la ingeniería inteligente (IE), no sólo conteniendo la relación de correspon- dencia de la función general, sino que también desarrolla: los grafos de red neutral, las reglas lógicas de red, su forma difusa, y los diagramas de relación, etc. Además de heredar el análisis de problemas y el procesamiento de la solución de la ingeniería de sistemas, también integra las ventajas de la inteligencia artificial, la tecnología de la computación inteligente (redes neuronales, lógica difusa y algoritmos genéticos, y otros), la teoría de la incertidumbre y la tecnología multi-agente. Proporcionando una forma y método para la investigación de la evolución compleja del sistema inteligente ( $\mathrm{Li}, \mathrm{Lin}, \& \mathrm{Hu}, 2013$ ).

Bajo el concepto de modelo generalizado, la IE utiliza la función establecida por tres elementos: So como el estado inicial, Sn estado objetivo y un conjunto de rutas inteligentes $\mathrm{PB}$ para describir la evolución del enorme complejo del sistema: S (So, Sn, PB).

La gráfica 1 refleja las diferencias de método entre la ingeniería de sistemas y la ingeniería inteligente. En la investigación científica, el sistema en ingeniería de sistemas consiste en un simple modelo matemático. Mientras que ingeniería inteligente, este tipo de desarrollo fácil-complejo-simple es también la encarnación del concepto "espiral ascendente de la negación de la negación" en la metodología científica.

Gráfica 1. Diferencias entre la ingeniería de sistemas e ingeniería inteligente

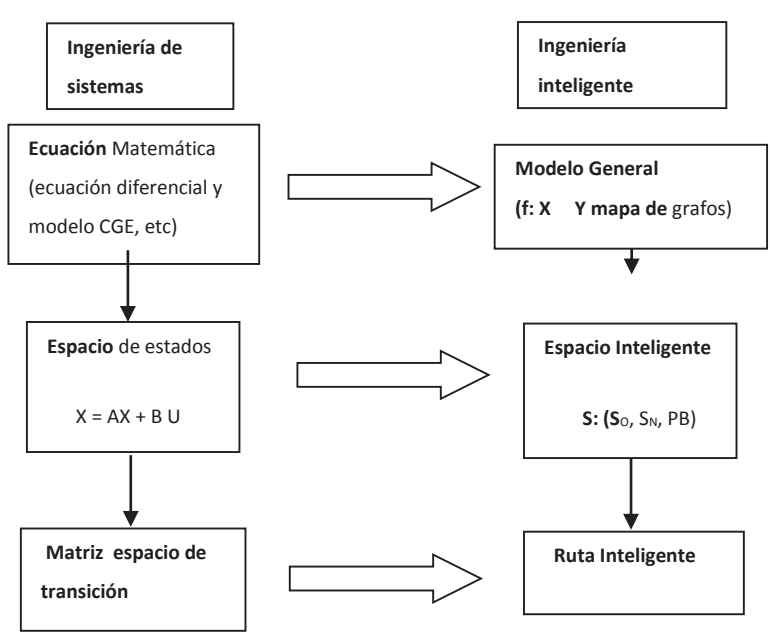

Fuente: Elaboración propia. 


\subsection{Tecnología de agentes}

Desde que John McCarthy fomento el concepto de agente en la década de 1950, la tecnología de agentes ha sido un componente importante de la investigación en el campo de la inteligencia artificial, también ha sido ampliamente investigado y aplicado en el análisis económico y la simulación de mercado de la energía.

Un agente es inicialmente una representación y en ocasiones se llama entidad colaboradora (Zou, Li, \& Yan, 2005). Es una entidad física o abstracta que puede actuar por sí mismo y con el medio, para hacer que reaccione ante los cambios del entorno. En cuanto a conocimiento, objetivo y capacidad. El conocimiento se refiere a la descripción del escenario en el cual el agente queda designado o en el problema que ha de ser resuelto por él; el objetivo representa las acciones tomadas por el agente que se orientan a una meta; y la capacidad significa que el agente tiene facultades para, la toma de decisiones, la planificación, y control.

En términos generales, un agente tiene cuatro características básicas: la autonomía, la reactividad, pro actividad y sociabilidad. La autonomía significa que un agente ha de controlar el poder de sus propios comportamientos o acciones y llevar a cabo su objetivo de forma independiente; la reactividad significa que un agente puede percibir el ambiente en el cual se mantiene y hace respuestas a los cambios del entorno en el tiempo; la pro actividad significa que un agente puede iniciar una acción de forma activa con el fin de lograr su objetivo; y la sociabilidad significa que un agente puede interactuar con otros agentes para llevar a cabo el objetivo de forma conjunta.

En un sentido más amplio, las entidades de comportamiento que tienen las características anteriores, como institución social, organismo, software, robot, y así sucesivamente, todos pueden ser tomados como agentes. Varios agentes forman un sistema multi-agente (MAS) en forma uniforme y coordinada integral, a través de la cooperación y la comunicación entre los agentes. Cada agente en el sistema resuelve problemas de forma independiente o en modo de cooperación.
En la actualidad, tras el establecimiento de la mayoría de los sistemas de agentes o MAS, el investigador no tendrá que intervenir necesariamente, sino que puede dejar que la interacción sea espontánea y evolucione por completo entre los agentes. El agente en este modelo de gestión de la demanda estará abierto; es decir, será un agente libre que se puede ajustar e intervenir por los expertos. Los principales aspectos intervenidos por los expertos incluyen principalmente el curso del agente en la evolución, las reglas de inferencia, el tiempo de funcionamiento y otros parámetros de ajuste del agente, etc.

\section{Estructura general de un sistema de gestión de la demanda eléctrica}

La estructura general incluye dos capas: la capa de soporte y la capa de aplicación.

\subsection{Funciones de la capa de aplicación}

En la parte de análisis del proyecto, se realizan las estadísticas de clasificación de los proyectos según su estado actual, en realización o finalizados, el avance de las condiciones de desarrollo por varios usuarios, las condiciones de aplicación de tecnología, así como la auditoría de consumo, conservación y ahorro de energía, el potencial de usuarios que utilizan el servicio, proporcionando una base fiable para el diseño de proyectos de DSM.

Análisis y predicción de carga: Analiza las condiciones potencia de carga, características y consumo de energía de los usuarios y pronóstico de la demanda de carga.

Evaluación de costo-beneficio: Evalúa el costo y el beneficio de los diferentes participantes del proyecto de DSM analizándolos, para generar las diversas medidas, de acuerdo con este método.

Simulación de políticas DSM: Simula los efectos de aplicación de las políticas pertenecientes al DSM y las influencias que puedan generar a partir de estas políticas que son investigados por cada interesado.

Evaluación integral del DSM: Evalúa a las empresas de generación de energía, empresas de la red 
Gráfica 2. Estructura de la función de software en la gestión de la demanda eléctrica

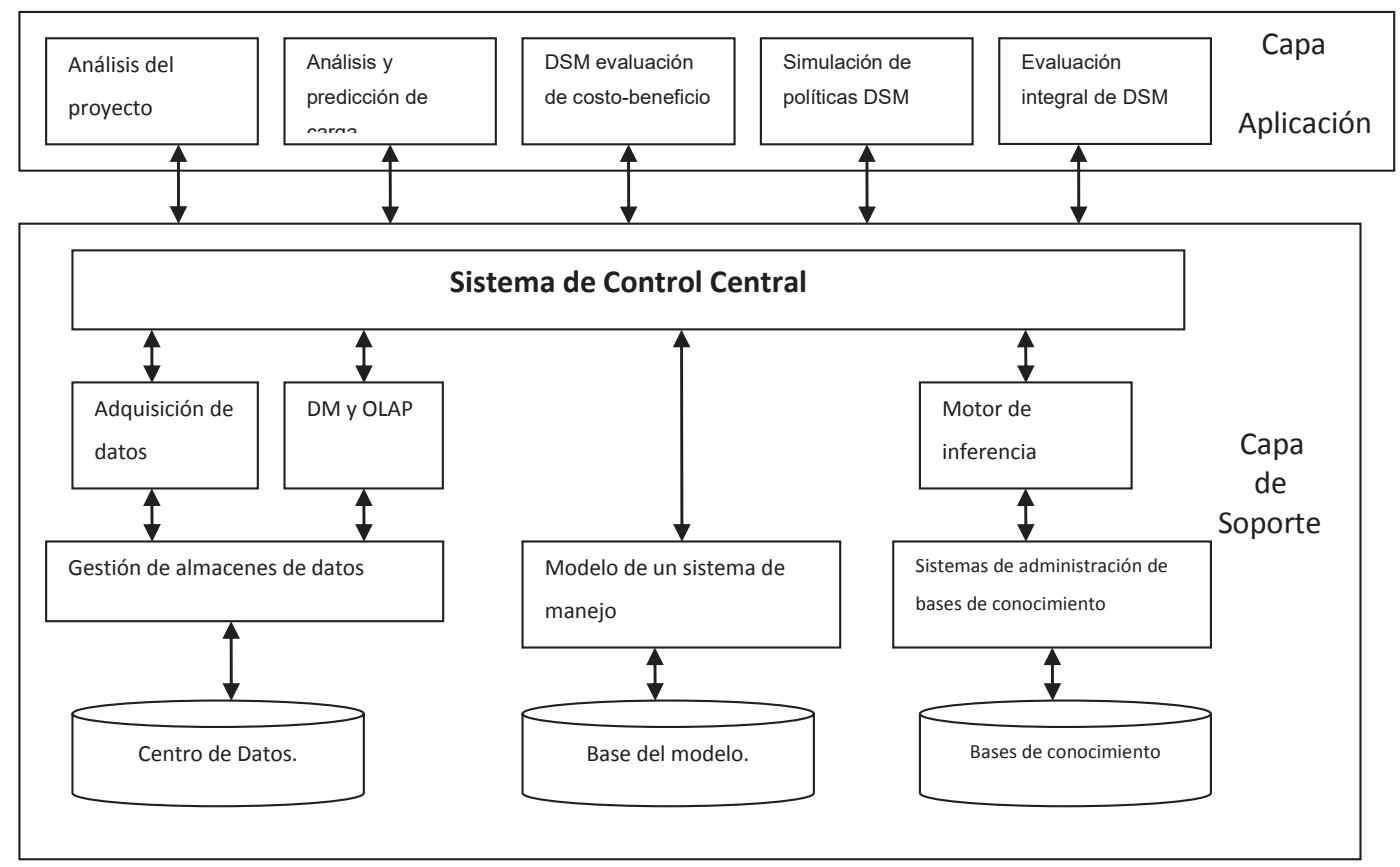

Fuente: Elaboración propia.

eléctrica, los usuarios de energía y el desarrollo social, entre otros aspectos que se consideran integralmente. Los métodos que se utilizan para evaluar los proyectos de DSM de forma integral, se utilizan para confirmar las ventajas y desventajas $y$ la viabilidad de los proyectos.

\subsection{Funciones de la capa de soporte del software}

Por su parte, en la capa de soporte están las funciones básicas necesarias para que el sistema pueda desarrollar la aplicación específica y se compone de diez componentes que son: un almacén o centro de datos (DW), una base de modelo y una base de conocimientos, la adquisición de datos, un motor de inferencia, la minería de datos $(\mathrm{DM})$, y procesamiento analítico en línea (OLAP) y un sistema de control central. El sistema de aplicación se refiere a los análisis de la aplicación del DSM y la experimentación que puede ser desarrollada por el sistema, incluyendo análisis de proyectos, análisis de la carga y la predicción, DSM de costo-beneficio evaluación, simulación de políticas DSM, y la evaluación integral de DSM, etc. (Schlenzig, 1998).

\subsection{Principales funciones de los componentes de la capa de soporte de software}

Un almacén de datos (DW) en un DSM se utiliza para almacenar una gran cantidad de datos, es de suma importancia que los datos relevantes se organicen en un conjunto de acuerdo a una cierta estructura. En general, la capacidad de un DW es muy grande; los datos están orientados a ciertos temas y se almacenan de acuerdo a una determinada estructura organizativa a conveniencia de su consulta y utilización.

El Sistema de gestión de DW: Trata de un conjunto de sistemas y programas que tienen funciones tales como el establecimiento del DW, supresión, modificación y mantenimiento, así como el almacenamiento de datos, recuperación, clasificación, índice, inventario, etc.

La adquisición de datos: Contiene la información relevante del DSM, incluyendo la información económica, la energía eléctrica, el clima, los productos de ahorro de energía, métricas de DSM, etc. Estas se ordenan y se recogen a través de múltiples for- 
mas que son pre-tratados y almacenados en la base de datos en diferentes tipos, de modo que sea posible llevar a cabo análisis precisos y cuantitativos de los problemas.

DM y OLAP: Son métodos y procesamiento de análisis de datos, basado en el DW. Se centra principalmente en la abstracción de información útil a partir de los datos existentes del DSM, como normas, conocimientos, experiencias, y así sucesivamente; el OLAP se concentra especialmente en la elaboración de diferentes tipos de análisis combinado y multidimensional de datos existentes, tales como análisis de datos de DSM en diferentes sectores como el tiempo, la región, la industria, etc.

Modelo de base: Es un complemento que almacena múltiples modelos de acuerdo a una determinada estructura organizativa en un ordenador, como los modelos de evaluación integral de análisis y estadística, predicción, planificación; y, estos modelos se pueden recombinar para nuevos modelos a fin de resolver problemas más complicados.

Sistema de gestión de bases de Modelo-Modelo: Se trata de un sistema o programa que controla el modelo de establecimiento, modificación, supresión, transferencia, consulta y evaluación de manera centralizada.

Base de conocimientos: Se trata de un aditamento, de conocimiento DSM y se componen de una serie de reglas, con expertos que lo complementan que tienen la experiencia y el conocimiento. Transferido por el motor de inferencia, se utiliza principalmente para resolver problemas semi - estructurados y no estructurados en la investigación de DSM.

Sistema de gestión de la base de conocimientos: Se trata de un sistema que controla la creación del conocimiento, su mantenimiento, transferencia, consulta y evaluación, y otros aspectos de una manera centralizada.

Motor de inferencia: Se trata de un sistema experto de tamaño pequeño que está conectado con la base de conocimientos y tiene la función de deduccióninducción.
Sistema de control central: Se trata de un módulo central del modelo de gestión de la demanda y es un puente que conecta la capa de aplicación, con la capa de soporte, que se utiliza para la coordinación y transferencia de todos los componentes de la capa de soporte. Con el fin de lograr la aplicación del DSM específico y reflejar el resultado a la aplicación capa.

\section{Modelo de evaluación DSM integral}

Como se muestra en la gráfica 3, el modelo de evaluación integral DSM incluye tres partes: configuración del índice de evaluación, cálculo del peso de índices y la evaluación de proyectos de DSM (Gu et al., 2012).

\subsection{Configuración del índice de evaluación}

Se refiere principalmente al establecimiento de un sistema de índices de evaluación integral del DSM, que incluye cuatro elementos: los índices de evaluación de la empresa de generación de energía, de la red eléctrica, de servicios de conservación y usuarios avanzados (ESCO) y los índices de evaluación de beneficios sociales; estos índices pueden ampliarse y completarse de forma continua según los requerimientos.

Los índices de evaluación de la empresa generadora de energía están representados en: la capacidad para evitar pico de carga, el costo del combustible, arranque de la unidad defectuosa, el costo de cierre, costo de operación y mantenimiento de la unidad, y los gastos de compensación por la contaminación ambiental, etc.

Los índices de evaluación de la red eléctrica se circunscriben esencialmente en: la reducción de ingresos, en la venta de energía, el costo de promoción de entrada, costo de inversión red de energía, la fiabilidad de alimentación, índice de satisfacción del usuario, la mejora del factor de carga, entre otros.

Los índices de evaluación ESCO y usuario avanzado incluyen entre otros: el costo total de inversión del equipo inicial, el costo adicional de operación y mantenimiento, el costo promedio de recuperación de la inversión, la compensación obtenida a partir 
Gráfica 3. Modelo de evaluación integral DSM

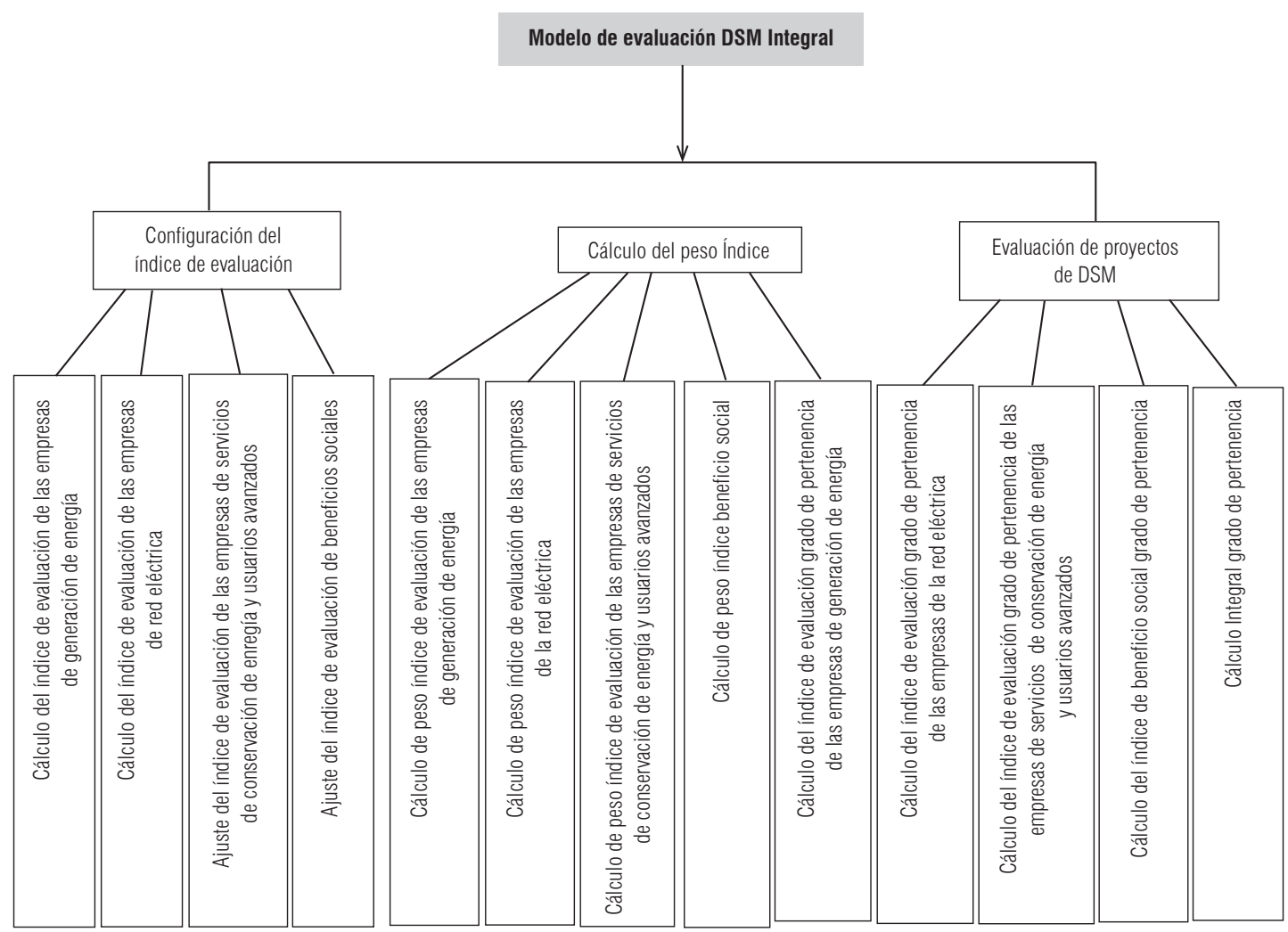

Fuente: Elaboración propia.

de la implementación de proyectos de DSM, y los gastos de reducción de electricidad, etc.

Los índices de evaluación de beneficios sociales toman en cuenta: la reducción del consumo de carbón para la generación de energía, la reducción de las emisiones de $\mathrm{CO} 2$, la reducción de las emisiones de $\mathrm{SO} 2$, y la reducción de las emisiones de NOX, etc.

\subsection{Cálculos de peso}

El cálculo de peso (Índice) es una parte importante de la evaluación integral. El índice de peso se refiere al grado de importancia de evaluación en relación con el objetivo de evaluación. Hay muchos métodos para el cálculo del peso de dicho índice, para ello se puede establecer por medio de análisis de proceso jerárquico (AHP).

La metodología del Proceso Analítico Jerárquico consiste en tres etapas:
Modelización, valoración y priorización. Entre los métodos de obtención de las prioridades locales destacan el método del autovector principal por la derecha (EGVM) propuesto por Saaty (1980) en la versión original de AHP y el método de la media geométrica por filas (RGMM), cuya utilización se ha incrementado significativamente en los últimos tiempos.

Gráfica 4. Estructura del índice AHP

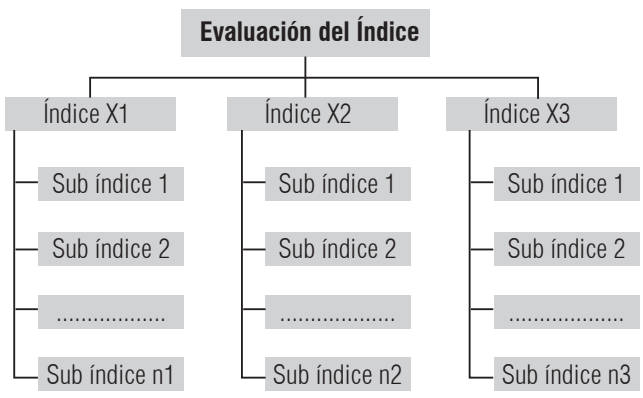

Fuente: Elaboración propia. 
Los principales pasos al emplear el método AHP para calcular el peso índice se dividen en: establecer la estructura jerárquica, formar la matriz de comparación pares de juicio, calcular el peso relativo de cada índice, inspección de consistencia de la matriz juicio y cálculo de peso global.

Supongamos que el sistema de evaluación de índice es una estructura jerárquica independiente. Como se muestra en la tabla 1 , el número de índices (o atributos) de cada capa generalmente no excede a 9.

Para formar la matriz de comparación pares de juicio. Se configura según la experiencia de los expertos, en referencia a los criterios de la Tabla 1.1 y comparando los índices de evaluación apareadas, la matriz de juicio A [refiriéndose a la fórmula (1.1)] se forma, entre los cuales, $a_{i j}$ indica la asignación de subíndice $U_{i j}(i=1,2, \ldots, n)$ en relación con el grado de importancia del subíndice bajo un mismo índice precursor.

$$
\mathrm{A}=\left[\begin{array}{ccc}
\mathrm{a} 11 & \cdots & a 1 m \\
\vdots & \ddots & \vdots \\
a m 1 & \cdots & a m m
\end{array}\right]
$$

Tabla 1. Referencia proporcional de la escala de calificación AHP

Según la comparación entre dos índices, es igual a

\begin{tabular}{|c|l|}
\hline 1. & Si están en el mismo grado de importancia \\
\hline 3. & Si el primero es un poco más importante que el segundo \\
\hline 5. & Si el primero es, evidentemente, más importante que el segundo \\
\hline 7. & Si el primero es fuertemente más importante que este último \\
\hline 9. & Si el primero es extremadamente más importante que este último \\
\hline $2,4,6$, y 8 & Si la condición entre las dos sentencias son contiguas \\
\hline Reciproco & $\begin{array}{l}\text { El último es más importante que la anterior. La escala de } \\
\text { comparación experto del índice xi y xj y la escala de compa- } \\
\text { ración de xi y xj son recíprocos entre sí. }\end{array}$ \\
\hline
\end{tabular}

Fuente: Elaboración propia.

La condición entre las dos sentencias adyacentes Cálculo del peso vector $\mathrm{W}$. El cálculo del vector de peso $\mathrm{W}$ muestra, la importancia de la ordenación de los índices de evaluación. La regla de cálculo se muestra en la Tabla 2.
Tabla 2. Proceso de cálculo de vector de peso

\begin{tabular}{|c|c|c|c|}
\hline $\begin{array}{c}\text { Matriz de juicio } \\
\text { A }\end{array}$ & $\begin{array}{l}\text { Producto } \\
\text { continuo } \\
\text { de } \\
\text { elementos } \\
\text { en cada } \\
\text { fila }\end{array}$ & $\begin{array}{c}\text { La raíz } \\
\text { m-ésima } \\
\text { producto } \\
\text { fila del } \\
\text { elemento }\end{array}$ & $\begin{array}{c}\text { Wi tiene que } \\
\text { llevar a cabo el } \\
\text { procesamiento } \\
\text { de } \\
\text { normalización } \\
\text { para } \overrightarrow{W l}\end{array}$ \\
\hline$\left[\begin{array}{ccc}\mathrm{a} 11 & \cdots & a 1 m \\
\vdots & \ddots & \vdots \\
\mathrm{am1} & \cdots & \mathrm{amm}\end{array}\right]$ & $M_{i}=\prod_{j=1} a_{i j}$ & $\overrightarrow{\omega \iota}=\sqrt[m]{M_{i}}$ & $\omega_{i}=\frac{\overrightarrow{\omega t}}{\sum_{i=1}^{m} \overrightarrow{\omega t}}$ \\
\hline
\end{tabular}

Observación es el número de orden de la matriz de juicio; Aij es el elemento de la matriz de juicio; Wi es el elemento i-ésimo del vector peso W

Fuente: Elaboración propia.

Inspección de consistencia de la matriz de juicio. Siguiendo los pasos a saber

i. Calcular la raíz máxima característica de la matriz de juicio.

$$
\lambda_{\max }=\sum_{i=1}^{m} \frac{(A . W)_{i}}{n \omega_{i}} \text { (1.3) }
$$

En la fórmula (1.3), A es la matriz de juicio; $W$ es el vector de peso; (A.W) indica el elemento i-ésimo de la matriz compuesta $A . W$, después de multiplicar la matriz $\mathrm{A}$ por $\mathrm{W}$; $\mathrm{m}$ es el número de orden de la matriz de juicio; $\omega_{i}$ i es el elemento i-ésimo del vector peso W.

ii. Calcular el índice de consistencia de desviación (CI) de la matriz de juicio.

Saaty sugiere para el método AHP convencional (en el que se utiliza el método del autovector principal para obtener las prioridades), que la inconsistencia sea capturada por un único, que refleja la desviación de los juicios aij con respecto al cociente estimado entre las prioridades, wi/wj. La medida de la consistencia propuesta se denomina Índice de Consistencia (CI) y se define como:

$$
C I=\frac{\lambda_{\max }-m}{m-1}(1.5)
$$

IA es el índice de consistencia aleatoria de A, es el índice de consistencia de una matriz de compara- 
ciones pareadas generada en forma aleatoria. Se puede mostrar que el IA depende del número de elementos que se comparan, y asume los siguientes valores:

Tabla 3. Índice de consistencia media al azar (IA)

\begin{tabular}{|l|c|c|c|c|c|c|c|c|c|}
\hline $\begin{array}{l}\text { El número } \\
\text { de orden de } \\
\text { la matriz de } \\
\text { juicio (m) }\end{array}$ & 1 & 2 & 3 & 4 & 5 & 6 & 7 & 8 & 9 \\
\hline $\begin{array}{l}\text { Índice de } \\
\text { consistencia } \\
\text { al azar (RI) }\end{array}$ & 0.00 & 0.00 & 0.58 & 0.90 & 1.12 & 1.24 & 1.32 & 1.41 & 1.45 \\
\hline
\end{tabular}

Fuente: Elaboración propia.

iii. Se calcula la razón de consistencia ( $\mathrm{RC}$ ) (o $\mathrm{CR}$, de Consistency Ratio).

Esta razón o cociente está diseñada de manera que los valores que exceden de 0.10 son señal de juicios inconsistentes; es probable que en estos casos el tomador de decisiones desee reconsiderar y modificar los valores originales de la matriz de comparaciones pareadas. Se considera que los valores de la razón de consistencia de 0.10 o menos son señal de un nivel razonable de consistencia en las comparaciones pareadas.

$$
C R=\frac{C I}{R I}
$$

\section{iv. Resultado consensuado}

Una vez realizada la totalidad de comparaciones se obtiene el resultado final consensuado y el ordenamiento de las alternativas. Este resultado está basado entonces, en las prioridades, en la emisión de juicios y evaluación hecha a través de las comparaciones de los componentes del modelo jerárquico, llevada a cabo por los actores.

\subsection{Evaluación de proyectos DSM}

El método de evaluación integral, empleado acá se utiliza para evaluar un proyecto de DSM. En primer lugar, se utiliza el grado de pertenencia para cada índice (se refiere al grado en que el índice de evaluación pertenece a un conjunto de evaluación, tales como muy buena, buena, comparativamente malos y otros) en el proyecto de DSM se corrobora de acuerdo a la opinión de expertos o en las condiciones de investigación requeridas; en segundo lugar, el grado de pertenencia integral del proyecto DSM se calcula mediante la combinación del peso de cada índice, y si el proyecto es viable DSM, y otros puede ser juzgado por el grado de pertenencia integral. El proceso de cálculo en relación con el método de evaluación integral fuzzy.

\subsubsection{Proceso de cálculo del método de evaluación integral difuso}

Método de evaluación integral difusa es uno de los métodos de evaluación integral (Han, Su, Yuan \& Wang, 2010), que incluye principalmente 5 partes: establecer el índice de evaluación, confirmación del peso índice, grado evaluación, la matriz de juicio confuso y calcular el grado de pertinencia integral.

El método de confirmación del índice peso es similar al empleado en el método AHP. De esta manera:

i. Criterios de evaluación establecidos.

Para la evaluación se establece que la función $\mathrm{V}=$ $\{v 1, v 2, v 3, \ldots, v k\}$ indica el grado de valoración. Por lo general, se utilizan cinco grados: $V=\{v 1$ (excelente), v2 (bueno), v3 (usual), v4 (pobre),..., vk (peor)\}

ii. Criterios de la matriz de juicio difuso.

Supongamos que el índice i de la capa inferior es Ui, el grado de pertenencia de los proyectos de DSM para selección en función $\operatorname{Vj}(j=1,2, \ldots, 5)(j=1$, $2, \ldots, 5)$, centrándose en ui es ry; de esta manera, el factor único conjunto de evaluación de ui se puede resolver: $\mathrm{Ri}=($ ri1, ri2, ri3, ri14, ri5)

De esta manera, el sistema de evaluación de índices $\mathrm{m}$ formarán una matriz de evaluación general R. Es decir, cada objeto evaluado confirma a la relación difusa $\mathrm{R}$ de $\mathrm{U}$ a U. En una matriz:

$$
R=\left(r_{i j}\right)_{m * 5}=\left[\begin{array}{ccc}
r_{11} & \cdots & r_{15} \\
\vdots & \ddots & \vdots \\
r_{m 1} & \cdots & r_{m 5}
\end{array}\right] \text { (1.7) }
$$


iii. Cálculo de grado de pertenencia integral.

El grado de pertenencia integral $\mathrm{B}$ de un proyecto de DSM puede calcularse mediante la combinación del vector de peso integral y el de AHP, que puede ser llamado sistema de evaluación integral del proyecto DSM.

$$
B=Y \cdot R=\left(b_{1}, b_{2}, b_{3}, b_{4}, b_{5}\right)
$$

En esta fórmula b1 indica que el proyecto de DSM es en grado de excelencia; b2 indica que el proyecto de DSM es en buen grado; b3 indica que el proyecto de DSM en el grado de general; b4 indica que el proyecto de DSM es en grado comparativamente malo; b5 indica que el proyecto de DSM es de mal grado. A través de este tipo de información, podemos juzgar si el proyecto DSM es factible.

\section{Conclusiones}

El modelo de gestión de la demanda es un sistema integrado compuesto de una red informática de la información, software, hardware, expertos de la industria, y personal técnico especializado, teniendo la plataforma de información DSM como base y el desarrollo de las investigaciones pertinentes, la promoción y el trabajo de aplicación en el campo del DSM hacia los gobiernos, las empresas de la red eléctrica, empresas de generación de energía, empresas de servicios de ahorro de energía, usuarios avanzados, etc.

A través del modelo de gestión de la demanda, se pueden modelar las condiciones de consumo de energía de los usuarios, la gestión de la demanda eléctrica y la información relevante de instituciones como: los gobiernos, los servicios públicos de red de energía y empresas de servicios de ahorro de energía, precios de venta y condiciones de aplicación de los diferentes productos de DSM, la información de diversos proyectos DSM implementados y las políticas, leyes y regulaciones, y otras informaciones pueden ser consultados en cualquier momento.

La evaluación de la viabilidad de varios proyectos de DSM puede llevarse a cabo para implementar el diseño, ejecución y desarrollo de proyectos de DSM que pueden ser monitoreados y así llevar a cabo la evaluación ex post de los proyectos; el análisis de simulación puede realizarse para ser aplicado en las políticas y medidas de DSM a conveniencia del interesado.

\section{Referencias}

Boyle, S. (1996). DSM progress and lessons in the global context. Energy Policy, 24(4), 345-359. doi:10.1016/03014215(95)00142-5.

Brañas-Garza, P. \& Espinosa, M. P. (2011). Experimental and economy behavior. Papeles Del Psicologo, 32(2), 185-193.

Chen, H., Wang, X., Wang, X. \& Chen, H. (2004). Design, implementation and application of a Java-based platform for electricity market auction experiments. Dianli Xitong Zidonghua/ Automation of Electric Power Systems, 28(17), 22-26, 44.

Elshkaki, A., Van Der Voet, E., Van Holderbeke, M. \& Timmermans, $\mathrm{V}$. (2004). The environmental and economic consequences of the developments of lead stocks in the Dutch economic system. Resources, Conservation and Recycling, 42(2), 133-154. doi:10.1016/j.resconrec.2004.02.008.

Gu, D., Liang, C., Zhong, J., Wang, J., Lu, W., Song, J. \& Wu, Y. (2012). Intelligent information acquisition and utilization in safety evaluation decision making process of power generation enterprises (Vol. 102 LNBIP).

Hamdan, H. A., Ghajar, R. F. \& Chedid, R. B. (2012). A simulation model for reliability-based appraisal of an energy policy: The case of Lebanon. Energy Policy, 45, 293-303. doi:10.1016/j. enpol.2012.02.034.

Han, Y., Su, G., Yuan, H. \& Wang, W. (2010). A collaborative early warning method of transportation during snow disaster based on fuzzy comprehensive evaluation. En Proceedings - 20107 th International Conference on Fuzzy Systems and Knowledge Discovery, FSKD 2010 (Vol. 2, pp. 945-951). doi:10.1109/ FSKD.2010.5569141.

Huang, J., Xue, Y., Wen, F., Xu, J., Xue, F. \& Dong, Z. (2011). A review of power market simulation platform. Dianli Xitong Zidonghua/Automation of Electric Power Systems, 35(9), 6-13, 35.

Li, Q., Lin, P. \& Hu, Y. (2013). Innovation and application of scientific research strategy to super high arch dam. Shuili Fadian Xuebao/Journal of Hydroelectric Engineering, 32(5), 281-287.

Räsänen, M., Ruusunen, J. \& Hämäläinen, R. P. (1995). Customer level analysis of dynamic pricing experiments using consumptionpattern models. Energy, 20(9), 897-906.

Schlenzig, C. (1998). Energy planning and environmental management with the information and decision support system MESAP. International Journal of Global Energy Issues, 12(1-6), 81-91.

Wei, Y.-M. \& Liang, Q.-M. (2009). A new approach to energy modelling: The se3T system and its multi-objective integrated methodology. International Journal of Global Energy Issues, 31(1), 88-109. doi:10.1504/IJGEI.2009.021544.

Zou, B., Li, Q.-H. \& Yan, M.-S. (2005). Agent-based simulation model on pool-based electricity market using locational marginal price. Zhongguo Dianji Gongcheng Xuebao/Proceedings of the Chinese Society of Electrical Engineering, 25(15), 7-11. 